\title{
Tratamiento combinado del adenoma prostático con el KPT-láser de 80 Watt y la resección transuretral de baja presión
}

\author{
Verger-Kuhnke AB, Reuter M, Epple W, Ungemach G, Beccaría ML. \\ Reuter Klink, Servicio de Urología del Hospital Karl-Olga, Universidad de Ulm, Stuttgart Alemania.
}

Actas Urol Esp 2006; 30 (4): 394-401

\section{RESUMEN}

TRATAMIENTO COMBINADO DEL ADENOMA PROSTÁTICO CON EL KPT-LÁSER DE 80 WATT Y LA RESECCIÓN TRANSURETRAL DE BAJA PRESIÓN

Introducción: La finalidad de este trabajo prospectivo es determinar la eficacia del método para la vaporización y eliminación del adenoma prostático. Se analizan también los resultados que se obtienen al combinar el método KTP-láser de 80 watt con la RTUP de baja presión.

Material y Método: 151 pacientes con HBP sintomática fueron tratados entre agosto del 2004 y enero del 2006 con el KTP-Láser o combinado con RUTP a baja presión. La resección adicional fue efectuada en aquellos pacientes con adenomas grandes o por tener el lóbulo medio acentuado. El efecto de la vaporización fue controlado al final de la operación por medio de TRUS (sonografia prostática transrectal).

Resultados: 151 pacientes fueron tratados en 2 grupos. El grupo 1 (n:43) aquellos con sólo tratamiento láser, sobre todo pequeños adenomas y el grupo 2 (n:108) con tratamiento combinado de KTPláser y RTUP a baja presión en adenomas grandes.

La flujometría máxima demuestra una mejoría del 65,6\% en el grupo 1 entre antes y después del tratamiento, en el grupo 2 la diferecia es de 122,4\%.

Conclusión: Nuestro estudio pone de manifesto, las ventajas de combinar ambos procedimientos quirúrgicos para obtener un mejor resultado en vaporización del adenoma prostático sobre todo en próstatas de gran tamaño.

Palabras clave: KTP-láser, RTUP, HPB, TRUS, flujometría máxima, tratamiento combinado.

\section{ABSTRACT}

\section{COMBINED TREATMENT OF PROSTATATIC ADENOMA WITH THE 80 WATT KTP-LASER} AND THE TRANSURETRAL RESECTION OF LOW PRESSURE

Introduction: The purpose of this prospective study is to determine the effectiveness of method for vaporisation and elimination of the prostate adenoma. The results are analysed that obtain when combining this Laser-method with the Resection of low pressure.

Material and Method: 151 patients with BPH symptomatic were treated between august 2004 and january 2006 with the KTP-Laser or combined with low pressure TURP. The additional Resection was carried out in those patients with great adenomas or to have accentuated middle lobule. The ablative effect was controlled at the end of the operation with TRUS (transrectal ultrasound).

Results: 151 patients were divided in 2 groups, group 1 (n:43) those with single laser treatment and group 2 (n:108) with combined treatment. The control of the post-miccional peak-flow demonstrates an increase of 65,6\% in group 1, and of $122,4 \%$ in group 2.

Conclusion: Our study puts of open, the advantages to combine both operative procedures to mainly obtain a better result in the ablation of the prostate adenoma in prostates of great size. 
$\mathrm{L}$ a historia del láser comenzó en 1917, cuando Albert Einstein demostró que era posible estimular los átomos de manera que todos ellos emitan luz con la misma longitud de onda, este proceso se conoce como emisión estimulada. La sigla LASER significa, amplificación de la luz por emisión estimulada de radiación. El rayo láser no es más que un rayo de luz de un único color amplificado.

En 1958, los físicos Arthur Schawlow y Charles Hard Townes describieron a grandes rasgos los principios del funcionamiento del láser. Años después apareció el primer rayo láser a partir de un cristal de rubí. En urología se utilizan el Nd:YAG láser, la enucleación prostática con Holmium láser (HoLEP) o el Low-power KTP láser (20-40 Watt) $3,4,8,9$. Todos ellos con propiedades hemostáticas semejantes a la RTUP, pero relativa escasa capacidad de vaporización del tejido prostático.

El último desarrollo técnico para el tratamiento láser de la HPB sintomática lo constituye el KTP (Kalium-Titanyl-Phosphat) 80 Watt "green Láser"1,2,5,11,12,13.

La finalidad de este trabajo prospectivo es determinar la eficacia del método para la vaporización y por consiguiente eliminación del adenoma prostático. Por otro lado se analizan los resultados que se obtienen al combinar dicho método con la RTUP de baja presión.

\section{MATERIAL Y METODO}

\section{Pacientes}

Entre Agosto del 2004 y Enero del 2006 se efectuó tratamiento endoscópico video-asistido con el KTP-Láser o combinado con RTUP en 151 pacientes con HPB sintomática. La indicación de combinar el láser con la RTUP a baja presión quedó a criterio de cada uno de los cinco urólogos que participaron en el estudio. La edad media de los pacientes fue de 71,6 años (55-85). 29 pacientes $(19,2 \%)$ tenían en el momento de la operación un catéter uretral o suprapúbico por padecer una retención aguda de orina o presentar una vejiga hipotónica con un residuo postmiccional mayor a $400 \mathrm{ml}, 149$ pacientes $(98,6 \%)$ fueron operados con un trócar suprapúbico.

Una RTUP adicional fue efectuada en aquellos pacientes con adenomas grandes o por tener el lóbulo medio acentuado. El efecto de la vaporización fue controlado al final de la operación por medio de TRUS (sonografia prostática transrectal).

Como estudios pre-operatorios se efectuaron: anamnesis, exploración física incluida la palpación prostática, evaluación de la calidad de vida mediante los cuestionarios EORTC QLQ-C30 y PR 25, medición del PSA, TRUS, flujometría urinaria, residuo post miccional y por último un pielograma endovenoso.

Se realizaron controles post operatorios antes del alta, a las 6 semanas a los 3 y 6 meses.

Técnica quirúrgica y propiedades fisicas del "green laser"

La operación se efectúa vía transuretral videoasistida a través de un instrumento $28 \mathrm{Ch}$. con una óptica de $25^{\circ}$. Nosotros empleamos un trócar suprapúbico para la irrigación continua de baja presión, que se coloca una vez llenada la vejiga con aproximadamente $450 \mathrm{ml}$ de líquido de irrigación.

La técnica quirúrgica se puede dividir en tres pasos: Primero se comienza con el KTP-láser; de esta forma se elimina la mayor parte del adenoma. Se prosigue a continuación con la RTUP, efectuada en 108 pacientes, (71,5\%).para eliminar los restos estromales irregulares en forma de una malla coraliforme blanco grisácea y así "alisar" la celda prostática y obtener material de la cápsula para biopsiar. Por último, y para obtener una coagulación óptima de la celda se aplica el KTP-láser por las excelentes propiedades hemostáticas del mismo ${ }^{4}$.

Al final de la operación se procede con un lavado vesical sobre el catéter suprapúbico y uretral.

Horas después o a más tardar al día siguiente, se retira el catéter uretral. Para comprobar la micción espontánea se procede a cerrar el catéter suprapúbico, lo que permite el llenado vesical espontáneo; una vez conseguida la micción, y con un residuo post-miccional menor a $100 \mathrm{ml}$ se retira dicho catéter y el paciente puede ser dado de alta (Fig. 1).

La fibra láser tiene un diámetro aproximado de 1,2 mm, con un haz de luz que permite dirigir exactamente el rayo láser, la distancia óptima de tratamiento es de 1 a $2 \mathrm{~mm}$ (técnica sin contacto). 


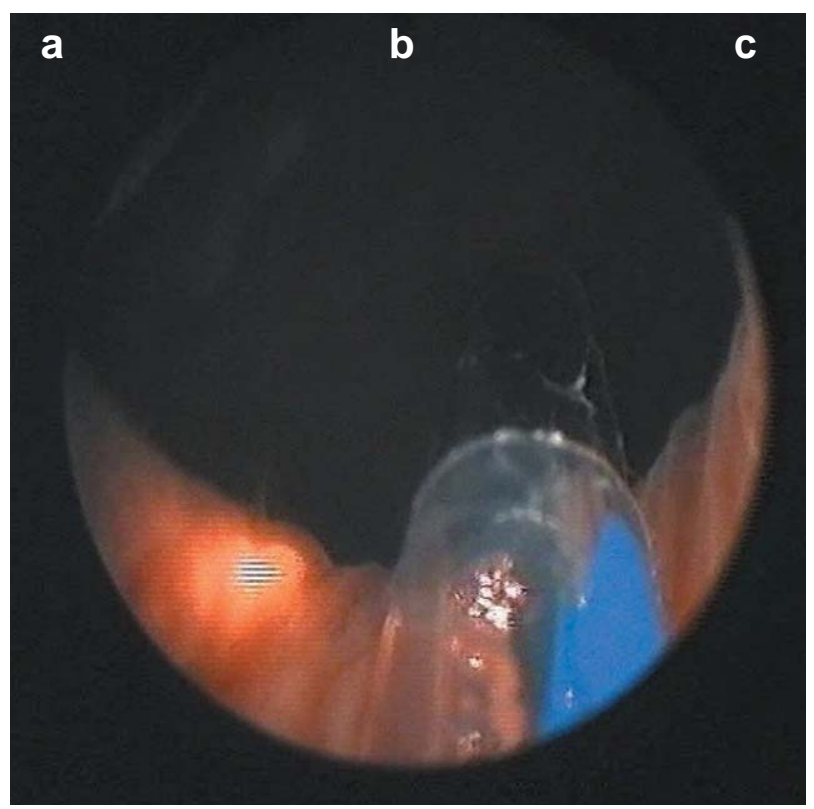

FIGURA 1.

a) Se observa la fibra láser, el cuello vesical y el haz del rayo láser.

b) Se aprecia el láser en acción con la vaporización del tejido prostático y la formación de burbujas.

c) Se observa el efecto ablativo inmediato luego del tratamiento (los restos estromales irregulares son visibles).

El "green láser" tiene una longitud de onda de $532 \mathrm{~nm}$, por lo que se encuentra dentro del espectro de la luz verde.

Una propiedad importante, es la reducida absorción de energía por parte del agua, lo que se traduce en una marcada vaporización citoplasmática superficial con formación de burbujas y una mínima difusión de calor y coagulación en los tejidos profundos, más allá de los 2 $\mathrm{mm}$ (poca penetración). Por otra parte, con esa longitud de onda se produce una absorción máxima de energía por parte de la hemoglobina, lo que se conoce como foto-selectividad; de esta manera se produce la vaporización del tejido adenomatoso de forma inmediata. El tejido estromal residual que se observa como una formación coraliforme impide un correcto reconocimiento de los límites entre el adenoma y la cápsula prostática (zona periférica); debido a este fenómeno es de utili- dad el control sonográfico transrectal intraoperatorio. El KTP-láser es de tipo pulsátil.

La RTUP se utiliza para asegurar la vaporización completa del tumor, sobre todo en adenomas de mas de $20 \mathrm{~g}$ (volumen prostático en la sonografia transrectal de mas de $40 \mathrm{ml}$ ).

\section{RESULTADOS (valores promedio)}

1. Edad del los pacientes 71,6 A. $(55-85$ A.)

2. Volumen prostático por TRUS: $\quad 50,4 \mathrm{ml}(18-150 \mathrm{ml})$

3. PSA

4. Tiempo quirúrgico

5. Energía total aplicada $3,8 \mathrm{ng} / \mathrm{ml}(0,34-15,9 \mathrm{ng} / \mathrm{ml})$ 70,3 Min. (25-165Min) 130,5 KJ (34-375 KJ)

6. Anestesia espinal en

149 Pac. $(98,6 \%)$

7. Anestesia general por anticoagulación con heparina en

2 Pac. $(1,3 \%)$

8. Riesgo quirúrgico según la sociedad americana de anestesiologia, ASA: Riesgo quirúrgico bajo, ASA I Riesgo quirúrgico intermedio, ASA II 97 Pac. $(64,4 \%)$ Riesgo quirúrgico alto, ASA III 29 Pac. $(19,2 \%)$

9. TURP adicional en 108 Pac. $(71,5 \%)$ con $24,5 \mathrm{~g}$ de adenoma resecado (1- $95 \mathrm{~g}$ ).

10. Histopatologia: 141 Pac. $(94,1 \%)$ con HPB, 10 Pac. (5,9 \%) con cáncer de Próstata (pTlG2).

11. Estadía hospitalaria 4,5 días (1-10).

12. No se observaron complicaciones intra operatorias. Transfusión de sangre en 1 paciente anticuagulado con cumarina (2 conservas). En 29 pacientes (19,2\%) se retiro el catéter uretral el mismo día, en 86 pacientes (56,9 $\%)$ al día siguiente y 22 (14,5\%) tuvieron el catéter mas tiempo y 14 pacienten $(9,3 \%)$ fueron dados de alta con catéter suprapúbico.

Parámetros urodinámicos (valores medios).

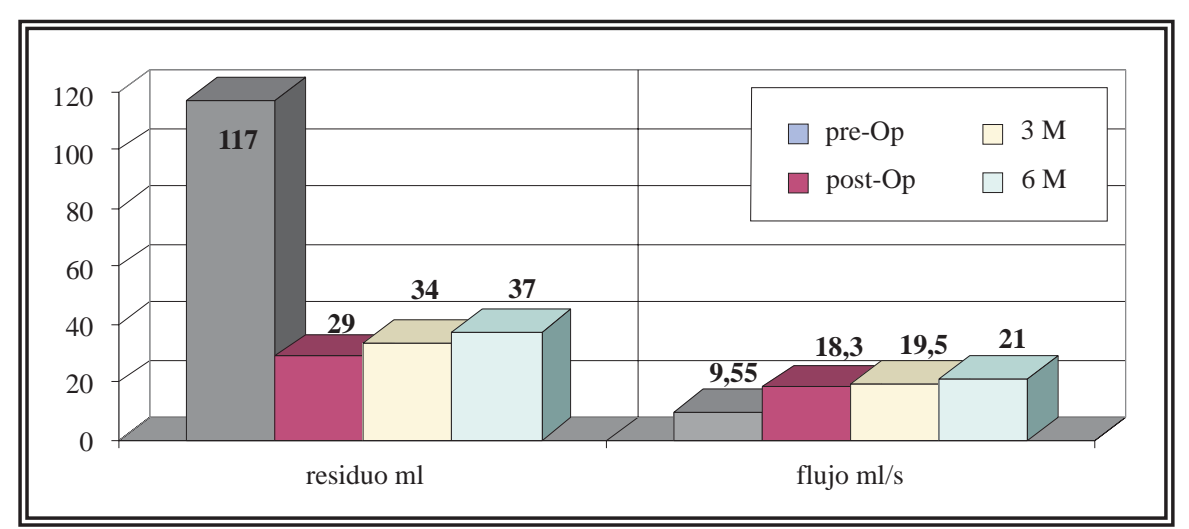

FIGURA 2. Residuo post-miccional en $\mathrm{ml}$ y flujo miccional máximo en $\mathrm{ml} / \mathrm{s}$, pre y post-operativo, a los 3 y 6 meses. 


\section{División de los Resultados en 2 grupos}

(valores medios) (Figs. 3 y 4) (Tabla 1)

Grupo 1: pacientes que solo recibieron tratamiento KTP-láser.

Grupo 2: pacientes que recibieron tratamiento combinado (KTP-láser y RTUP).

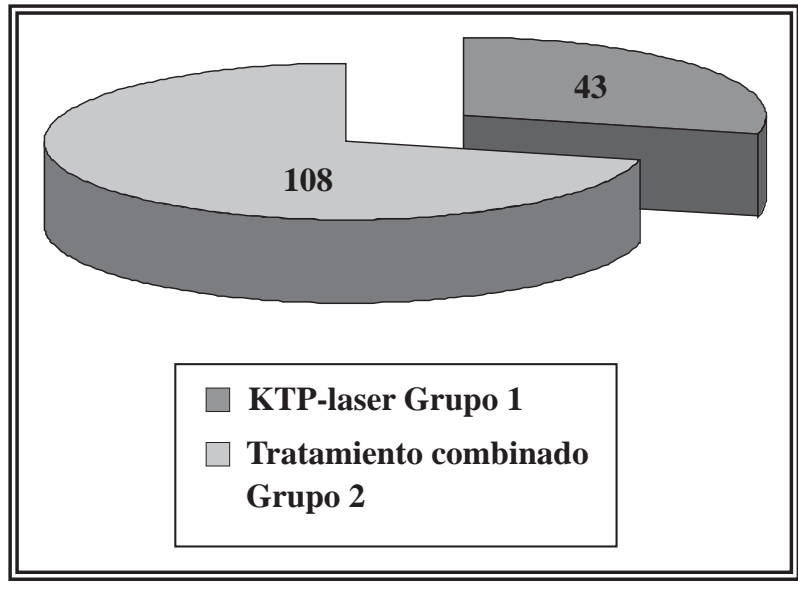

FIGURA 3. División de los pacientes en 2 grupos.

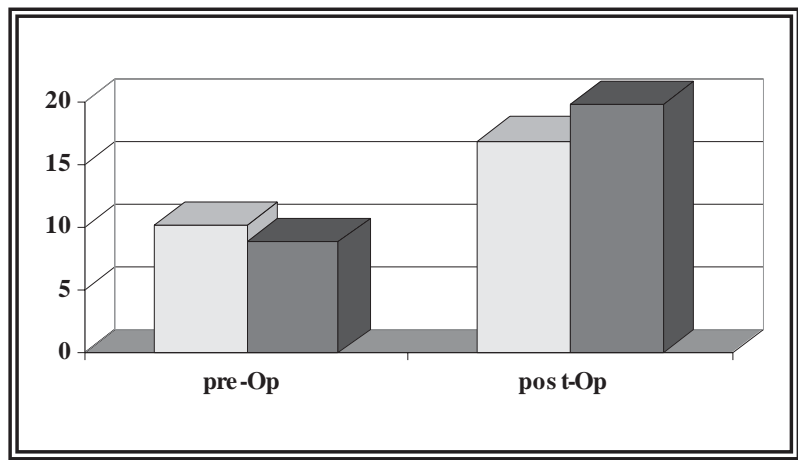

FIGURA 4. Flujometría miccional máxima en ambos grupos en $\mathrm{ml} / \mathrm{sec}$ (columna gris claro grupo 1 y columna gris oscuro grupo 2).

Tabla 1

Volumen prostático transrectal (TRUS) pre y post operativo en los 2 grupos

\begin{tabular}{llcc}
\hline TRUS & & $\begin{array}{c}\text { Volumen } \\
\text { abladido }\end{array}$ & $\begin{array}{c}\text { Porcentaje } \\
\text { de tejido } \\
\text { abladido }\end{array}$ \\
\hline Pre-op Grupo 1 & $40,5 \mathrm{ml}$ & & \\
Post-op Grupo 1 & $25,7 \mathrm{ml}$ & $14,8 \mathrm{ml}$ & $36,5 \%$ \\
Pre-op Grupo 2 & $60,3 \mathrm{ml}$ & & \\
Post-op Grupo 2 & $22,1 \mathrm{ml}$ & $38,2 \mathrm{ml}$ & $63,4 \%$ \\
\hline
\end{tabular}

En las Figuras 5 y 6 se observan los resultados obtenidos antes y después de la operación con KTP-láser. El volumen prostático con TRUS varía de aprox. $39 \mathrm{ml}$ antes de la operación a 29 ml después. También se aprecia el efecto de la vaporización, el tiempo quirírgico fue de 60 minutos y la energía aplicada de $133 \mathrm{KJ}$.

En las Figuras 7 y 8 se observan los resultados en un caso de tratamiento combinado de KTP-Láser y RTUP. El volumen prostático con TRUS varia de aprox. $50 \mathrm{ml}$ antes a $15 \mathrm{ml}$ después del tratamiento. La energía láser aplicada fue de $68 \mathrm{KJ}$ y se resecaron adicionalmente $5 \mathrm{~g}$ de adenoma. El tiempo quirúrgico fue de 80 minutos. Se puede apreciar claramente la vaporización completa del adenoma.

En las Figuras 9 y 10 se presenta un caso de tratamiento combinado (KTP-láser+RTUP) con un adenoma de aprox. $105 \mathrm{ml}$ Se aplicaron $238 \mathrm{KJ}$ de energía, se resecaron adicionalmente $66 \mathrm{~g}$ de adenoma en un tiempo quirúrgico de 165 minutos. El resultado es la vaporización completa del adenoma, pudiendose observar solamente la zona periférica en el control post-operatorio de aprox. $14 \mathrm{ml}$.

Resultados en la calidad de vida:

La calidad de vida fue objetivada con los formularios de EORTC QLQ-C30 y PR25 (Genitourinary Group of the European Organisation for Research and Treatment of Cancer).

La escala es de 1 a 7 puntos, siendo 1 una calidad de vida mala y 7 sobresaliente.

La calidad de vida de los pacientes del grupo 1 (solamente KTP-láser) fue antes de la operación en promedio de 4,5 puntos y a los 3 meses de 5,3 puntos. De estos pacientes, 50\% mantuvieron el mismo valor en el periodo comprendido entre antes de la operación y a los 3 meses de la misma, 45\% lo mejoró y sólo el 5\% sufrió desmejoría.

Por otro lado, la calidad de vida de los pacientes del grupo 2 (KTP-láser + RTU-P) fue antes de la operación de 4,3, a los 3 meses de 5,4 de los cuales el $40 \%$ de los pacientes mantuvieron el valor, el $40 \%$ lo mejoro y $20 \%$ presento desmejoría en la calidad de vida.

En un estudio prospectivo sobre la calidad de vida en 202 pacientes que recibieron una RTU-P 


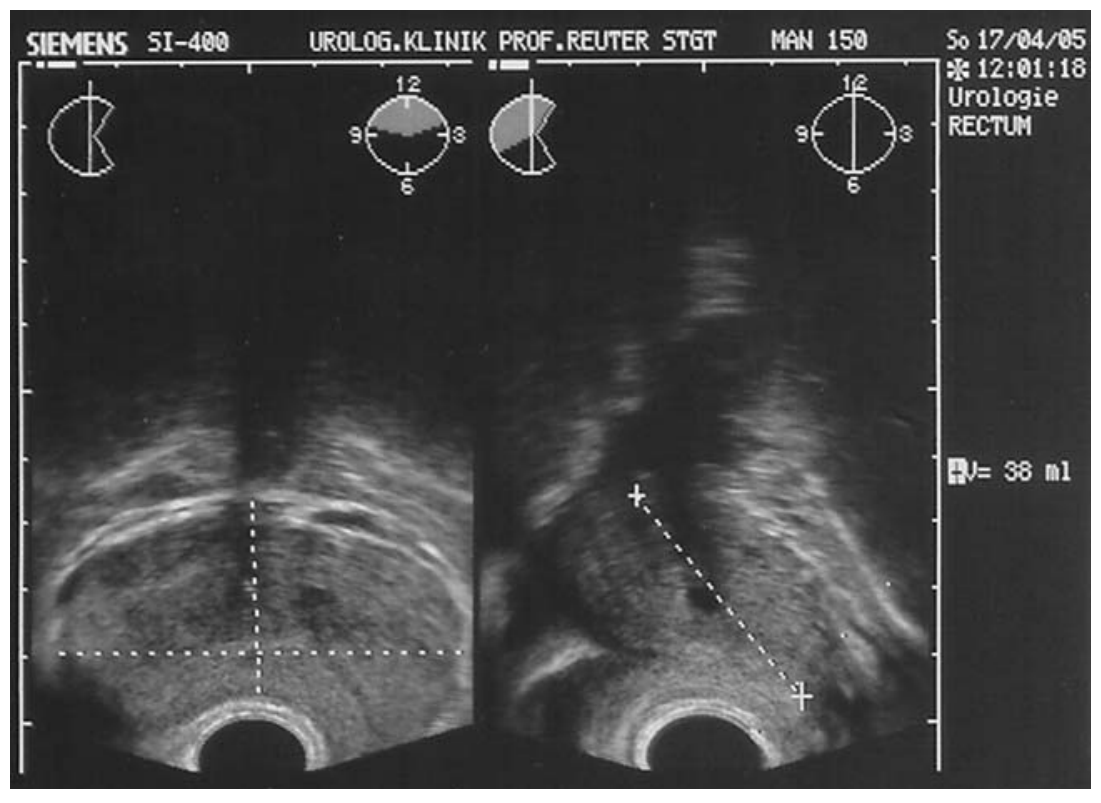

FIGURA 5. (Paciente grupo 1).

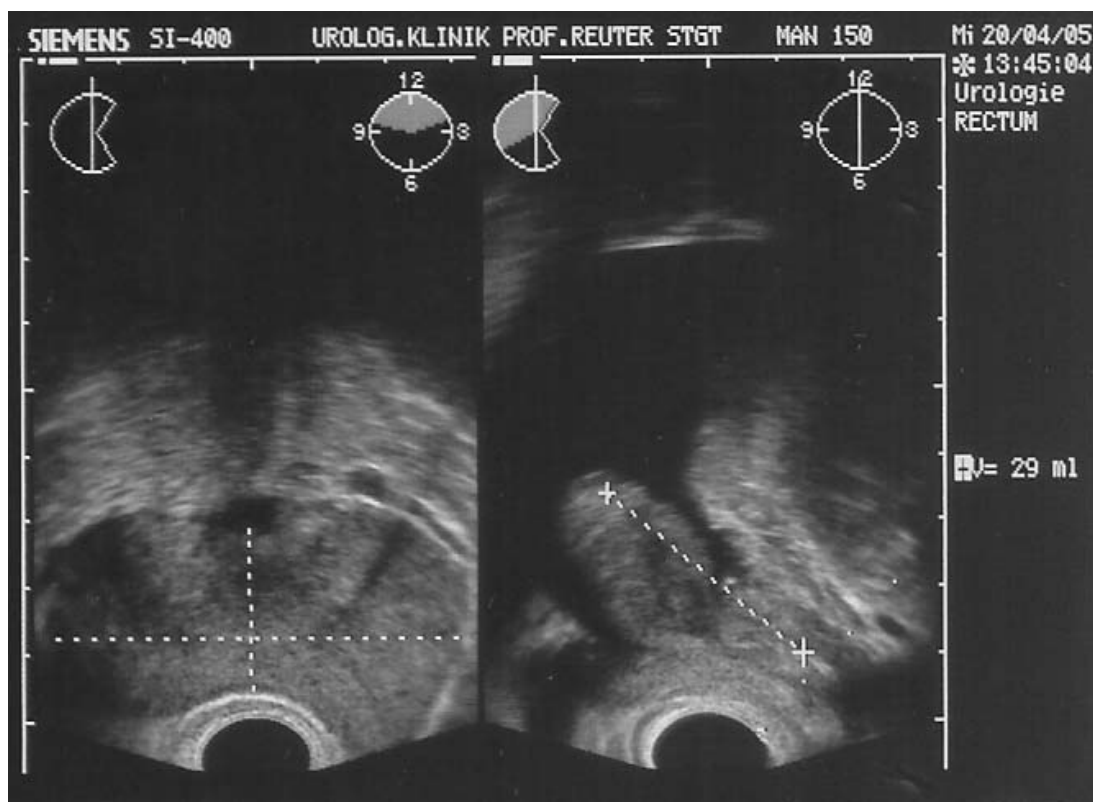

FIGURA 6

entre los años 2004-2005 en nuestro servicio de Urología se obtuvieron los siguientes resultados:

La calidad de vida antes de la operación fue de 4,7 a los 3 meses de 5,1. Los valores se mantuvieron en el 33\% de los pacientes, en el $35 \%$ mejoraron y en el 32 \% desmejoraron.

Como conclusión se destaca que los pacientes tratados con KTP-láser desmejoraron en la calidad de vida en hasta un $5 \%$, los pacientes que recibieron un tratamiento combinado desmejora- ron hasta un 20\%, mientras que los pacientes operados solamente con RTU-P desmejoraron hasta un $32 \%$ a los 3 meses (Tabla 2).

En el grupo 1 (sólo KTP-láser), se vaporizaron en promedio $14,8 \mathrm{ml}$ de tejido prostático en 60,5 minutos de tratamiento (controlado por TRUS), lo que representa un promedio de 1 ml vaporizado en 4,08 minutos.

Por otro lado en el grupo 2 (KTPláser+TURP), se vaporizaron un promedio de $38,2 \mathrm{ml}$ en $80,2 \mathrm{mi}$ nutos, lo que significa que por 1 $\mathrm{ml}$ de tejido resecado son necesarios 2,09 minutos.

Como conclución se descata que en el grupo con tratamiento combinado el tiempo quirúrgico empleado es aproximadamente la mitad del que seria necesario con unicamente KTP-láser.

\section{DISCUSIÓN}

En este estudio presentamos los resultados obtenidos en 151 pacientes con el tratamiento de KTPláser y RTUP. Los pacientes se dividieron en 2 grupos, grupo 1 sólo con tratamiento láser, (43 pacientes) y grupo 2 con tratamiento combinado, (108 pacientes).

Si observamos los resultados urodinámicos de los 2 grupos pre y post operatorios, observamos un notable aumento del flujo entre ambos a favor de los que recibieron tratamiento combinado (65,6\% KTP sólo, 122,4\% KTP-láser+RTU).

En el grupo 1, tratado solamente con KTPláser se vaporizaron un promedio de14,8 $\mathrm{ml}$ de adenoma, y todos presentaban al final del tratamiento tejido adenomatoso restante (Fig. 5-6). En el grupo 2 se vaporizaron $38,2 \mathrm{ml}$ de tejido prostático y en el control ecográfico transrectal se observó solamente la zona periférica. El tiempo quirúrgico fue similar en los dos grupos pero con una diferencia de $20 \mathrm{ml}$ en el volumen prostático a favor del grupo 2 . 


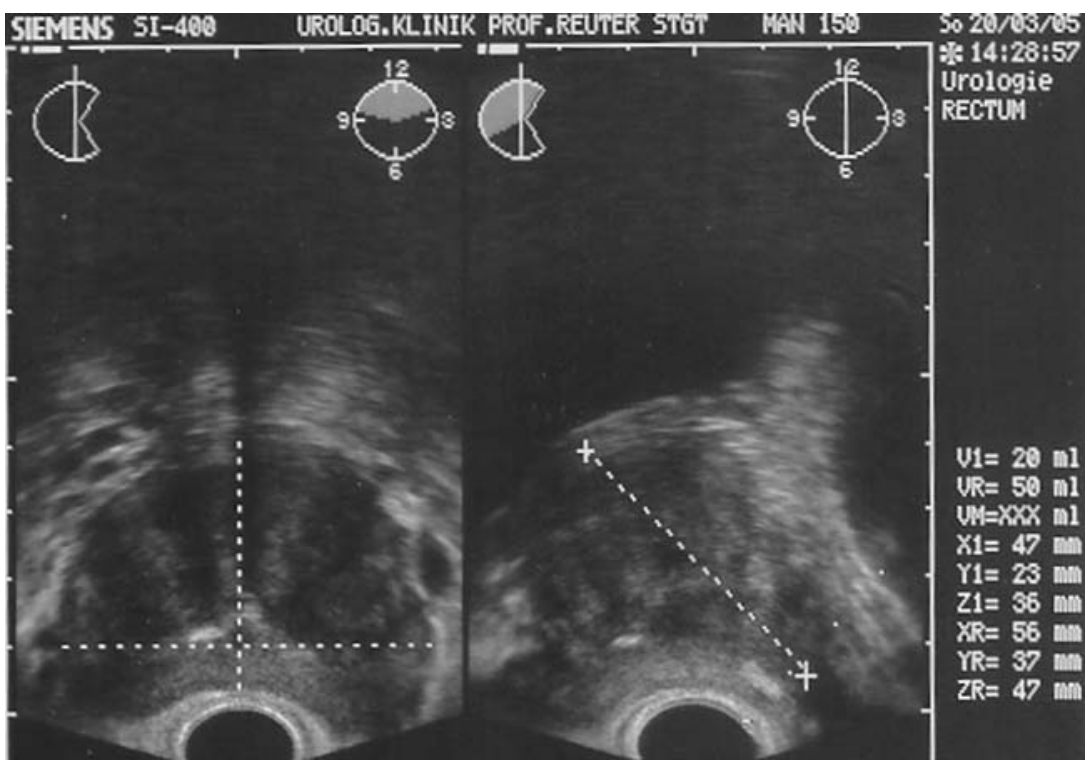

FIGURA 7. (Paciente grupo 2).

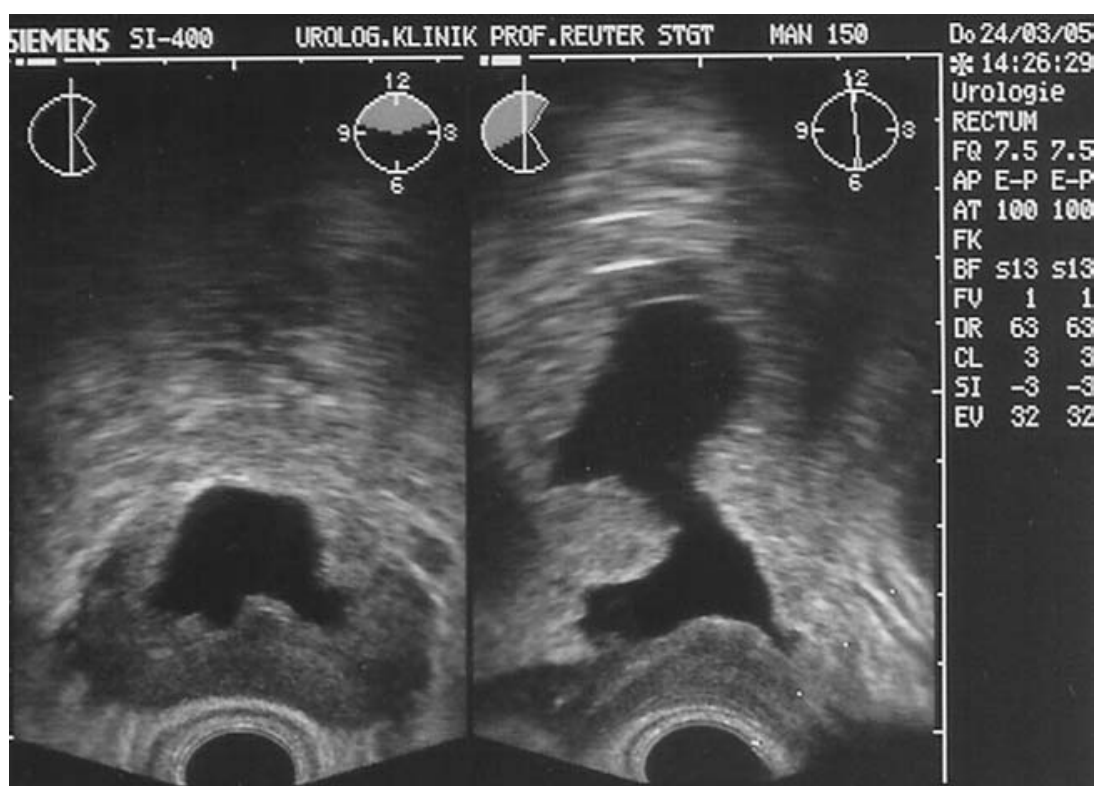

FIGURA 8

Si observamos los resultados obtenidos por medio de la RTUP, método considerado como el Standart de oro para el tratamiento de la HPB en un grupo de 1544 pacientes, con una edad promedio de 69,2 (41-94) operados en nuestra clínica, se resecaron en promedio 36,7 g (rango 5-160 g) $10,11,14$.

Si comparamos los resultados obtenidos con la RTUP de esos 1.544 pacientes con nuestro grupo 1 podemos concluir que con el KTP-láser no se obtienen en promedio dichos resultados, tanto en el tiempo quirúrgico como en la cantidad de adenoma vaporizado y tejido adenomatoso residual.

Lo que hay que mejorar son los endoscopios ya que no permiten una buena conducción de la sonda ni adecuada visibilidad en casos de sangrado de pequeñas arterias.

Con el KTP-láser no es posible obtener biopsias típicas del tejido prostático; en nuestra serie de pacientes que recibieron tratamiento combinado se pudieron diagnosticar 10 adenocarcinomas de la próstata (5,9\%), los cuales tenían un PSA por de bajo de $4 \mathrm{ng} / \mathrm{ml}$ y una palpación prostática normal ${ }^{7}$.

La estadía hospitalaria en promedio de pacientes que recibieron solamente TURP es de 7,5 días. Al aplicar el KTP-láser en el tratamiento, se consigue una reducción marcada de la hospitalización a 3,5 días (reducción del 53,3\%). Esto se puede explicar por el hecho de que la herida operatoria se estabiliza mas rápidamente y el sangrado es menor o nulo, lo que permite retirar los catéteres tanto uretrales como suprapúbicos de forma anticipada. No hemos tenido la necesidad de una coagulación de urgencia por sangrado en ninguno de los últimos casos.

Si se analizan los resultados obtenidos con los formularios de calidad de vida, antes de la operación y a los 3 meses se observa una mejoría similar en los dos grupos, lo que permite afirmar que la resección adicional no representó para los pacientes un factor negativo, a pesar de ser un tratamiento más agresivo que el láser.

\section{CONCLUSIONES}

La vaporización total del adenoma prostático, con el KTP-láser 80 Watt sólo es posible en próstatas de poco volumen y no mayores a $40 \mathrm{ml}$ en la TRUS. 


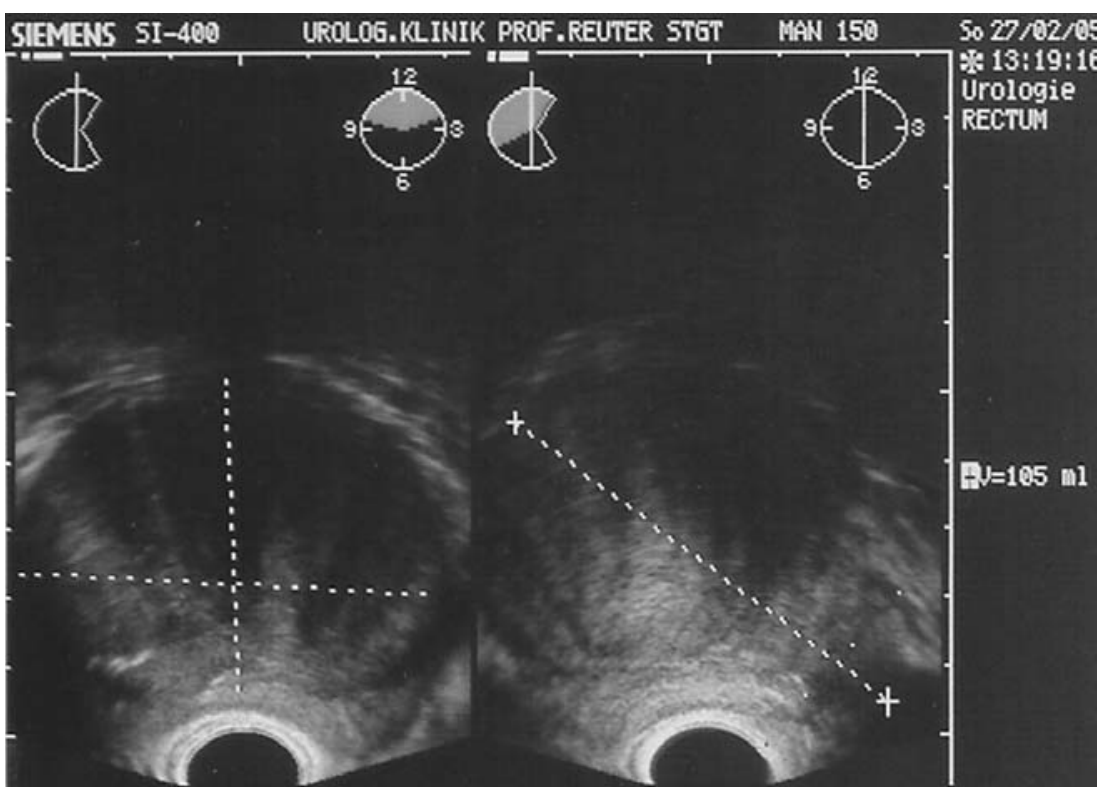

FIGURA 9. (Paciente grupo 2).

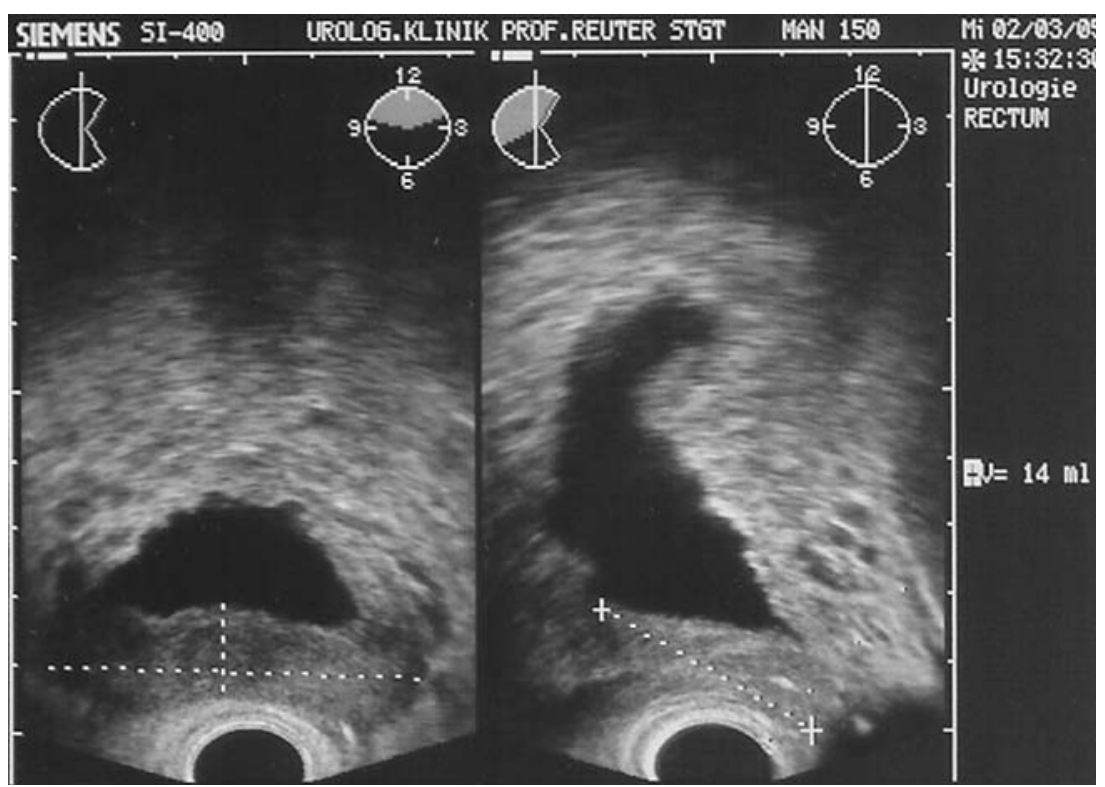

FIGURA 10

Tabla 2

Tiempo quirúrgico en ambos grupos

\begin{tabular}{lcccc}
\hline & Tiempo OP & $\begin{array}{c}\text { ml Tejido } \\
\text { abladido TRUS }\end{array}$ & $\begin{array}{c}\text { Tiempo para } \\
\text { abladir 1 ml }\end{array}$ & $\begin{array}{c}\text { Diferencia en } \\
\%\end{array}$ \\
\hline Grupo 1 & $60,5 \mathrm{~min}$. & $14,8 \mathrm{ml}$ & $4,08 \mathrm{~min}$. & \\
Grupo 2 & $80,2 \mathrm{~min}$. & $38,2 \mathrm{ml}$ & $2,09 \mathrm{~min}$ & $50 \%$ \\
\hline
\end{tabular}

El tiempo quirúrgico con el KTP-láser es sustancialmente mayor, y estimado en el doble que con la RTUP, para obtener un resultado final semejante.

El KTP-láser posee exelentes propiedades hemostáticas que lo hacen adecuado para el tratamiento de pacientes de alto riesgo quirúrgico. El método ofrece una alta seguridad y ninguna complicación intraoperatoria.

El KTP-láser produce en los pacientes durante las primeras semanas al post operatorio mayores sintomas irritativos que requieren tratamiento con alfabloqueantes o anticolinérgicos en combinación con fitofármacos (Cernilton).

Con el método láser no es posible obtener muestras típicas para la biopsia.

Si se pretende una vaporización completa del adenoma, también en próstatas grandes (más de $100 \mathrm{ml}$ ) se puede combinar el método KTP-láser con la RTUP de baja presión. De esta manera se adicionan los efectos positivos de ambos métodos; por un lado el mejor efecto hemostático del KTPláser, con una estancia hospitalaria reducida a la mitad y por otro lado la posibilidad de una eliminación completa del tumor con una mayor rapidez quirúrgica y la obtención de material para la histopatología ${ }^{6}$.

El control ecográfico transrectal intraoperatorio permite comprobar la vaporización completa del adenoma, lo que asegura un óptimo resultado quirúrgico.

\section{REFERENCIAS}

1. Bachmann A, Wyler A, Ruszat R, Gasser T, Sulser T. Photoselektive KTP-laser vaporisation of the prostate. First experience after 50 procedures. J Endourol 2003 (Supp 1):A 188. 
2. Bachmann A, Reich O, Wyler St, Ruszat R, Casella R, Gasser $\mathrm{T}$, et al. Urologische Klinik, Universität Basel und Urologische Klinik, Ludwig Maximilians Universität München. Die 80Watt-Kalium-Titanyl-Phosphat-(KTP)-Laser-vaporisation der Prostata. Der Urologe A Okt 2004(43):1262-1270.

3. Corica A, Marchiotto M, Verger-Kuhnke A, Anchelerguez R, Prats J. Urology Laser vaporisation of the prostate: experience in a selected group of patients. Departament, Cuyo University (Mendoza, gentina).Congreso Norte-Americano de Urologia A.U.A. 1997.

4. Gurdal M, Tekin A, Yucebas E, Sengor F. Departament of Urology, Haydarpasa Numune Education and Research Hospital, Istambul, Turkey.Nd:YAG laser ablation plus transurethral resection for large prostates in high-risk patients. Urology 2003 Nov;62(5):914-917.

5. Hai MA, Malek RS. Departament of Urology, Oakwood Annapolis Hospital, Wayne, Michigan, USA. Photoselective vaporisation of the prostate: initial experience with a new 80 W KTP laser for the treatment of benign prostate hyperplasia.J Endourol 2003 Mar;17(2):93-96.

6. Kitamura H, Masumori N, Tanuma Y, Yanase M, Itoh N, Takahashi A, et al. Departament of Urology, Sapporo Medical University School of Medicine, Japan. Does transurethral resection of the prostate facilitate detection of clinically significant prostate cancer that is missed with systematic sextant and transition zone biopsies? Int J Urol 2002 feb;9(2):95-99.

7. Kumar SM. From the Departament of Urology, Oakwood Annapolis Hospital, Wayne, Michigan, USA. Photoselective vaporisation of the prostate: a volume reduction analysis in patients with lower urinary tract symptoms secondary to benign prostate hyperplasia and carcinoma of the prostate. J Urol 2005 Feb;173(2):511-313.

8. Kuntz RM, Lehrich K, Ahyai S. Departament of Urology, Auguste Viktoria Hospital, Berlin, Germany. Transurethral holmium laser enucleation of the prostate compared with transvesical open rostatectomy:18.month follow-up of a randomised trial. L Endourol 2004 Mar;18(2):189-191.

9. Kuo RL, Kim SC, Lingeman JE, Paterson RF, Watkins SL, Simmons GR, et al. Methodist Hospital Institute for Kidney Stone Disease, Indiana University School of Medicine, 1801 North Senate Bouleward, Suite 220, Indianapolis, USA. Hol- mium laser enucleation of prostate (HoLEP): the Methodist Hospital experience with greater than 75 gram enucleations. J Urol 2003 Jul;170(1):149-152.

10. Litteljohn JO, Ghafar MA, Kang YM, Kaplan SA. Transurethral resection of the prostate: the new old standard. Curr Opin Urol 2002 12:19-23.

11. Reich O, Bachmann A, Schneede P, Zaak D, Sulser T, Hofstetter A. Departament of Urology, University Hospital Grosshadern, Ludwig Maximilians University of Munich, Germany. Experimental comparison of high power (80 W) potassium titanyl phosphate laser vaporisation and transurethral resection of the prostate. J Urol 2004 Jun;171 (6Pt):2502-2404.

12. Reich O, Bachmann A, Siebels M, Hofstetter A, Stief CG, Sulser T. Departament of Urology, University Hospital Grosshadern, Ludwig Maximilians University of Munich, Germany. High power (80W) potassium-titanyl-phosphate laser vaporisation of the prostate in 66 high risk patients. J Urol 2005 Jan;173(1):158-160.

13. Sandhu JS, Ng C, Vanderbrink BA, Egan C, Kaplan SA, Te AE. Departament of Urology, New York Presbyterian Hospital Cornell University Weill Medical College, New York, USA. High-power potassium-titanyl-phosphate photoselective laser vaporization of the prostate for treatment of benign prostate hyperplasia in a men with large prostates. Urology 2004 Dec;64(6):1155-1159.

14. Van Melick HH, Van Venrooij GE, Eckhardt MD, Boon TA. Departament of Urology, University Medical Center Utrecht, The Netherlands. A randomised controlled trial comparing transurethral resection of the prostate, contact laser prostatectomy and electrovaporisation in men with benign prostatic hyperplasia: urodynamic effects. J Urol 2002 Sep; 168(3): 10581062.

Dr. A.B. Verger-Kunhneke

Servicio de Urología

Hospital Karl-Olga

Hack Str. 61 - 70190 Alemania

E-Mail: andresbverger@aol.com

(Trabajo recibido el 16 de enero de 2006) 